\title{
La perspectiva de estudiantes universitarios sobre la escuela como promotora de salud y vida saludable
}

\section{The perspective of university students on the school as a promoter of health and healthy lifestyle}

\author{
Juan José Leiva Olivencia ${ }^{1}$, Antonio Matas Terrón ${ }^{2}$, Noelia Margarita Moreno Martínez ${ }^{3}$ \\ ${ }^{1}$ Didáctica y Organización Escolar, Universidad de Málaga, España (juanleiva@uma.es) \\ ${ }^{2}$ Métodos de Investigación y Diagnóstico en Educación, Universidad de Málaga, España (amatas@uma.es) \\ ${ }^{3}$ Teoría e Historia de la Educación, Universidad de Málaga, España (nmarg@uma.es)
}

\section{RESUMEN:}

Hablar hoy de educación es subrayar sus potencialidades para la promoción activa de hábitos de vida saludable, lo que constituye un eje relevante en la nueva configuración de funciones en las instituciones escolares del siglo XXI. En este artículo se ofrecen los resultados más relevantes de un estudio realizado en un contexto universitario con estudiantes de Grado de Educación Primaria. El objetivo del estudio ha sido conocer y comprender las actitudes y percepciones que tienen los estudiantes participantes acerca de la escuela como promotora de salud y hábitos de vida saludable. Para ello se ha empleado un cuestionario ad hoc. La muestra que formó parte del presente estudio ascendió a 115 sujetos. Los resultados indican que los estudiantes tienen una visión pedagógica positiva de la Educación para la Salud (EpS), sobre su relevancia en el marco de un currículum de carácter inclusivo. Ahora bien, se muestran críticos con el tratamiento didáctico de la $\mathrm{EpS}$, y es que son conscientes de la existencia de un escaso compromiso real del alumnado de desarrollar hábitos de vida saludables.

PALABRAS CLAVE: EDUCACIÓN PARA LA SALUD, VIDA SALUDABLE, BIENESTAR.

\begin{abstract}
:
Healthy Education is a basic dimension in the 21stcentury scholar functions. In this paper is shown the more highlighted outcomes from a research about understanding attitudes and perceptions from university students about the role of the school for boosting a healthy style of life. An ad-hoc questionnaire was applied to 115 students from the Faculty of Education of the University of Málaga. The main conclusion is student have a very positive image about the Education for Health, and its inclusion in the curriculum. However, they are critics about a real engagement from schools for developing healthy habits.
\end{abstract}

KEYWORDS: $\quad$ HEALTH $\quad$ EDUCATION,




\section{INTRODUCCIÓN}

La educación actual viene asumiendo funciones y responsabilidades de una enorme multiplicidad y complejidad de significados sociales y culturales, lo cual está siendo ya acogido y atendido por instituciones, administraciones y, sobre todo, centros escolares. En este punto, debemos hacer mención a la creación en noviembre de 2015, del Sello Vida Saludable, que surge como consecuencia de la publicación en BOE de la Orden ECD/2475/2015, de 19 de noviembre, por la que se crea el distintivo de calidad de centros docentes Sello Vida Saludable.

En este punto, cabe señalar que la Ley Orgánica 2/2006, de 3 de mayo, de Educación, señala en su artículo 2.1 que el sistema educativo español se orientará, entre otros, a la consecución de la adquisición de hábitos intelectuales y técnicas de trabajo, de conocimientos científicos, técnicos, humanísticos, históricos y artísticos, así como el desarrollo de hábitos saludables, el ejercicio físico y el deporte. Por tanto, valorar la higiene y la salud, además, constituye uno de los objetivos principales de la educación primaria, tal y como postula el artículo 17 de la mencionada Ley Orgánica 2/2006, de 21 de mayo, afianzándose tales hábitos de cuidado y salud corporales e incorporando la educación física y la práctica del deporte para favorecer el desarrollo personal y social a lo largo de la educación secundaria obligatoria.

De igual modo, la Ley Orgánica 8/2013, de 9 de diciembre, para la mejora de la calidad educativa, señala en su disposición adicional cuarta que las administraciones educativas adoptarán medidas para que la actividad física y la dieta equilibrada formen parte del comportamiento infantil y juvenil. El apartado 3 del artículo 43 de la Constitución Española liga la educación y la salud al instar a los poderes públicos a fomentar la educación sanitaria, la educación física y el deporte, facilitando asimismo la adecuada utilización del ocio.

La escasa actividad física y los hábitos no saludables o poco saludables durante la infancia y la juventud no solo son peligrosos para la salud, sino que conllevan una baja forma física y una menor autoestima, que en edad escolar deriva en un menor rendimiento académico y finalmente en el abandono educativo temprano.

Por ello, junto con la actividad física, la dieta sana, la higiene y el sueño regular y descanso adecuado son los cimientos para llegar a la vida adulta sanos y poder disfrutar de una vida plena. Es fundamental el vínculo, por tanto, que une a la educación con el necesario cuidado de la salud y con el hecho de disponer de hábitos conducentes a una vida sana. La salud proporciona, junto con la educación, las mejores oportunidades para que una sociedad tenga adecuados niveles de bienestar

Con el propósito de fomentar los hábitos que conduzcan al desarrollo de una vida saludable en todas sus vertientes, el Ministerio de Educación, Cultura y Deporte procedió mediante la orden ECD/2475/2015, de 19 de noviembre, a crear el distintivo de calidad de centros docentes Sello Vida Saludable con objeto de reconocer públicamente a aquellos centros docentes, tanto públicos como privados, que fomenten el aprendizaje de la salud en el ámbito educativo, así como la asunción de prácticas de vida saludable y una educación física que permita el adecuado desarrollo personal y social a lo largo de la escolarización de los alumnos.

Como afirman Cubero, Calderón, Costillo y Ruiz (2011) la EpS se encuentra en una nueva situación de interseccionalidad y confusión dentro de la docencia del Espacio Europeo de Educación Superior (EEES). Dada la demanda e interés que genera en el futuro profesional de la educación, pero con el "inconveniente de un bajo contenido curricular en la formación de estos futuros docentes, es ahora dentro del EEES donde posee una vía para progresar y mejorar dentro de los programas formativos de grado y máster, relacionados con la educación" (Cubero, Calderón, Costillo, y Ruiz, 2011, p. 51)

La EpS comprende las oportunidades de aprendizaje creadas conscientemente destinadas a mejorar la alfabetización sanitaria que incluye la mejora del conocimiento de la población y el desarrollo de habilidades personales que conduzcan a la mejora de la salud. Es un proceso educativo que tiene como finalidad responsabilizar a los ciudadanos en la defensa de la salud propia y colectiva. Es un instrumento de la promoción de salud y por tanto una función importante de los profesionales sanitarios, sociales y de la educación.

Ni que decir tiene que, en la promoción de la salud, como un nivel necesario de la atención integral, es imprescindible para una respuesta eficaz a los problemas de salud más relacionales y de origen social. Cuando se refiere a la EpS lo hace considerándola como un instrumento necesario para cada uno de los niveles de atención, también y sobre todo de la promoción de la salud de la que se sirve técnica y metodológicamente. En efecto, EpS como disciplina surge ante la necesidad de proporcionar a las personas los medios necesarios para mejorar la salud, a través de la modificación de conductas de 
riesgo. En la XXXVI Asamblea Mundial de la Salud se definió a la EpS como cualquier combinación de actividades de información y educación que lleve a una situación en la que las personas sepan cómo alcanzar niveles de salud óptimos y busquen ayuda cuando lo necesiten. La EpS tiene como objetivo primordial diseñar programas de intervención destinados a modificar creencias, costumbres y hábitos no saludables, además de promover, proteger y fomentar la salud.

La EpS como estrategia pedagógica en las escuelas $\mathrm{y}$, también, en los centros formativos superiores, contribuye a fomentar hábitos alimentarios saludables que contrarresten la prevalencia de obesidad a través de la promoción de hábitos alimentarios y estilos de vida saludables en los escolares, que inculque en los niños actitudes, conocimientos y hábitos saludables que favorezcan su crecimiento y desarrollo, el fomento de su salud y la prevención de las enfermedades evitables de su edad (Macías, Gordillo y Camacho, 2012).

En el contexto legislativo español son claras las referencias a la educación y promoción de salud. La Constitución, en su artículo 43, reconoce el derecho a la protección de la salud y establece que los poderes públicos fomentarán la EpS. La Ley 14/86 General de Sanidad en su artículo 6 declara, igualmente, que las actuaciones de las administraciones públicas sanitarias estarán orientadas a la promoción de la salud y a promover el interés individual, familiar y social por la salud mediante la adecuada educación sanitaria de la población.

Según González de Haro (2008) la salud es un valor pedagógico y social y debería ser, sin lugar a dudas, uno de los ejes centrales sobre el que se apoye el proceso educativo en estos tiempos de globalización cultural y de proliferación de estilos de vida poco saludables. Por tanto, la escuela se configura como el escenario ecológico privilegiado donde se desarrolla una función pedagógica con valores humanos de enorme importancia, por su importante contribución a la configuración de la persona en todas sus dimensiones vitales, tanto intrapersonales como interpersonales.

Los objetivos de la EpS en las instituciones escolares actuales deben modularse entre la construcción emergente de valores individuales y comunitarios de protección y empoderamiento de la salud como un bien trascendental en la vida de las personas. En este punto, tal y como viene recogido en Orden ECD/2475/2015, de 19 de noviembre, por la que se crea el distintivo de calidad de centros docentes Sello Vida Saludable, se puede destacar que los centros educativos deben promover la adquisición y logro de los siguientes objetivos institucionales en materia de promoción activa de la salud:

a) Fomentar el rol de la educación en hábitos saludables como una parte integrante de la educación integral, diseñando, a tales efectos, un programa de educación en hábitos saludables que comprenda la programación y establecimiento de objetivos educativos y de los instrumentos de evaluación.

b) Promover estilos y hábitos de vida saludables, a partir de las necesidades específicas del alumnado del centro docente, y especialmente en los ámbitos de la nutrición y alimentación saludables y del deporte contemplando el enfoque de género y la equidad para evitar desigualdades en la salud.

c) Fortalecer y mejorar los comportamientos saludables de los alumnos, con especial énfasis en la higiene y la prevención.

d) Afianzar los hábitos de cuidado y salud corporales e incorporar la educación física y la práctica del deporte para favorecer el desarrollo personal y social de los alumnos.

e) Fomentar en el alumnado la adquisición de capacidades y competencias que les permitan cuidar su cuerpo tanto a nivel físico como mental, así como valorar y tener una actuación crítica ante la información, publicidad y ante actitudes sociales que puedan repercutir negativamente en su desarrollo físico, social y psicológico.

f) Conocer y detectar las situaciones de riesgo para la salud, relacionadas especialmente con el consumo de sustancias con potencial adictivo, tanto de comercio legal como ilegal, así como con la ejecución de determinadas actividades que puedan generar comportamientos adictivos, con especial atención a la anorexia y a la bulimia, contrastando sus efectos nocivos y proponiendo medidas de prevención y control.

g) Promover la comprensión y valoración de la importancia de preservar el medio ambiente por las repercusiones que el mismo tiene sobre la salud de las personas.

h) Fomentar la responsabilidad del alumnado en las decisiones diarias del mismo, y el conocimiento de las consecuencias que las mismas tienen en su salud y en el entorno que les rodea. 
i) Promover el adecuado conocimiento de la salud e higiene sexual y reproductiva.

j) Promover la puesta en valor de la investigación, desarrollo e innovación en los avances médicos y en el impacto de la calidad de vida de las personas.

k) Promover la formación continua del personal docente que preste sus servicios en el centro, en materia de educación en hábitos saludables y en especial sobre alimentación saludable y práctica de actividad física.

Por su parte, Muñoz y Cabieses (2008, p. 139) consideran que

Las universidades desempeñan un papel protagonista en la sociedad donde están insertas. Orientadas al liderazgo y al desarrollo del conocimiento, son escenario de formación técnica y profesional de los miembros de la comunidad y, a través de la educación, la investigación y la difusión del saber, orientan y apoyan los cambios en diversos espacios nacionales e internacionales. Toda universidad cumple su misión social al penetrar en la cultura en que está inmersa, mediante el avance constante del saber en los temas de mayor relevancia y necesidad para cada país. De esta forma, las instituciones de educación superior buscan comprender los fenómenos y aportar soluciones que enriquezcan y orienten el futuro nacional, regional y mundial. En otras palabras, la existencia misma de una universidad implica su participación en la cultura y en los procesos relevantes de la nación a la que pertenece.

El carácter transversal e interdisciplinar que tiene la EpS desde un punto de vista didáctico supone un elemento de enorme potencialidad en el marco de un tratamiento educativo integral, favorecedora de una educación en valores proactiva en cualquier contexto educativo, incluida lógicamente la enseñanza superior (Gavidia, 2001).

En función de todo ello, el objetivo general de este estudio ha sido identificar las actitudes y percepciones que tienen los estudiantes de primer curso del Grado de Educación Primaria de la Facultad de Ciencias de la Educación de la Universidad de Málaga sobre la escuela como promotora de la salud y la vida saludable.

\subsection{Material y método}

Durante el curso académico 2016-2017 se ha desarrollado en la Facultad de Ciencias de la Educación de la Universidad de Málaga un estudio de investigación dirigido a los estudiantes del Grado de Educación Primaria, que constituyen el grupo más numeroso de estudiantes las distintas titulaciones que oferta la mencionada institución formativa. la muestra que formó parte del presente estudio ascendió a 115 sujetos, todos ellos de $1^{\circ}$ curso, sobre una población total de 410 (de $1^{\circ}$ curso de grado de Educación Primaria). La mayoría de la muestra es femenina, lo cual se puede generalizar a todo el grado. La edad media del alumnado es de 19 años.

Para la realización del presente estudio se procedió a la elaboración de un instrumento ad hoc, un cuestionario tipo Likert con veinte ítems, que fue validado por expertos. El instrumento se respondió anónimamente, pero además de las respuestas a los contenidos centrales del cuestionario, los participantes respondieron también algunos datos sociodemográficos personales, tales como el sexo y la edad.

Se procedió a la realización de un análisis estadístico de corte descriptivo básico en la medida en que una muestra más bien reducida no podía ser objeto de generalización, pero sí de poder subrayar ciertas tendencias en las percepciones y actitudes de jóvenes universitarios que estudian el Grado de Educación Primaria.

\subsection{Resultados}

El alumnado considera de forma mayoritaria, cerca de un $40 \%$ si sumamos las dos opciones favorables, que la escuela es un lugar privilegiado para el desarrollo de acciones educativas de EpS. Un 30,2\% del alumnado afirme que está poco de acuerdo con esta consideración (figura 1).

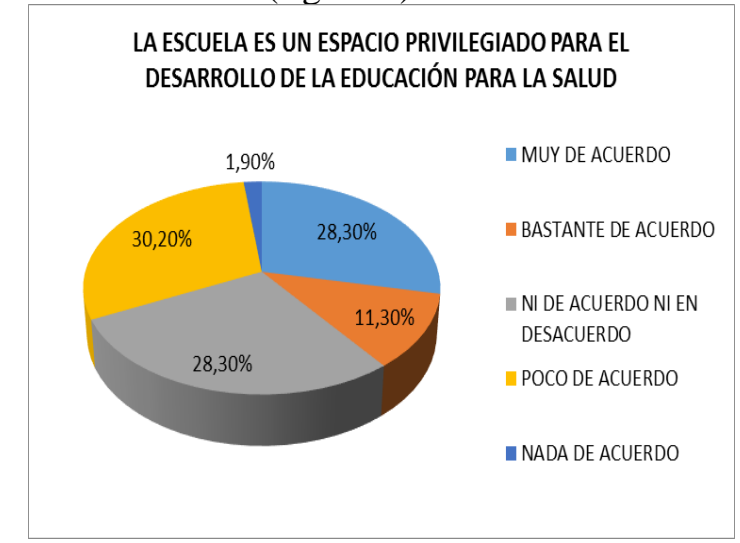

Figura 1. La escuela es un espacio privilegiado para el desarrollo de la EpS 
Más de la mitad de los encuestados plantea de forma clara su desacuerdo ante la afirmación de que la escuela se preocupe por el bienestar y la salud del alumnado más que por cuestiones académicas y de aprendizaje escolar. Se destaca que un $32,1 \%$ está nada de acuerdo con ello y que un $47,2 \%$ poco de acuerdo. Únicamente un $20,8 \%$ muestra una posición ambivalente sobre esta cuestión (figura 2).

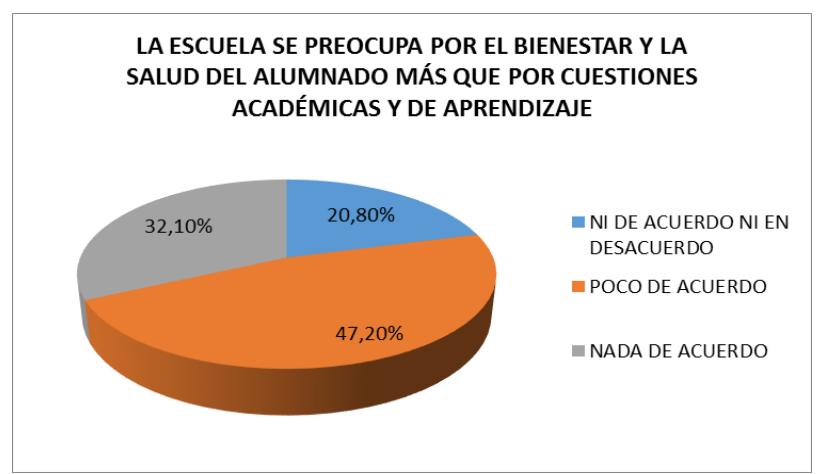

Figura 2. La escuela se preocupa por el bienestar y la salud del alumnado más que por cuestiones académicas y de aprendizaje

Una amplia mayoría de los estudiantes $(41,5 \%$ poco de acuerdo, y $35,8 \%$, nada de acuerdo) conciben la escuela como un espacio se cultivan actitudes y hábitos de vida saludables. Esto contrasta claramente con una minoría de estudiantes $(18,9 \%)$ que sí afirman que en el contexto educativo sí se haría un esfuerzo positivo por impulsar y generar hábitos de vida saludables (figura 3).

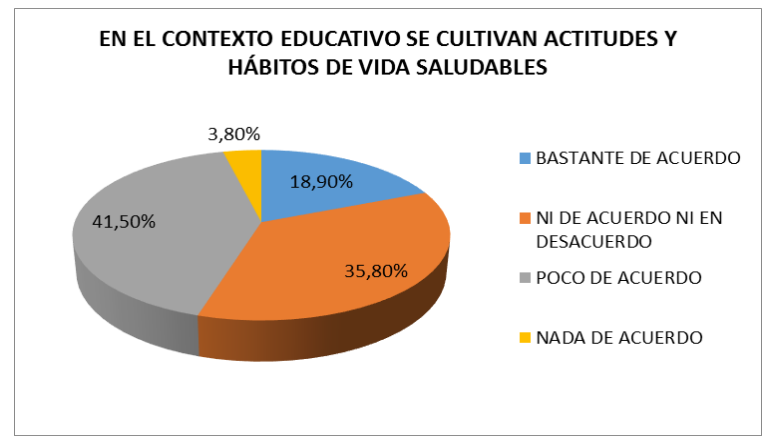

Figura 3. En el contexto educativo se cultivan actitudes y hábitos de vida saludables

La escasez de contenidos curriculares vinculados a la EpS es valorado negativamente por el alumnado universitario, y es que se percibe que existen pocos contenidos específicos en el currículo ordinario que puedan permitir un óptimo abordaje didáctico de iniciativas para la adquisición de hábitos de vida saludable y de calidad de vida. Un $30,2 \%$ y un $13,2 \%$ comparten esta idea. Frente a este posicionamiento, únicamente un $15,1 \%$ y un $3,8 \%$ valoran positivamente la capacidad de la escuela como espacio formativo donde sí se trabaja curricularmente la EpS (figura 4).

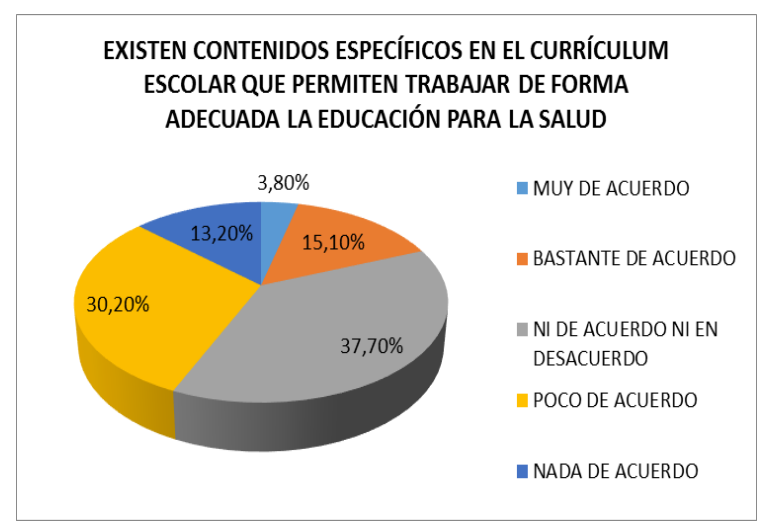

Figura 4. Existen contenidos específicos en el currículum escolar que permiten trabajar de forma adecuada la EpS

Otro aspecto del presente estudio trata del quehacer docente. Se encuentra una división nítida sobre aquellos estudiantes que valoran que el profesorado está preparado para trabajar adecuadamente la EpS, y, por ende, se trabajan de forma específica la relevancia de mantener una alimentación equilibrada, y quienes consideran que no existe ni ese compromiso ni esa acción continuada de trabajo docente en favor de la EpS. De forma específica, un $18,9 \%$ y un $37,7 \%$ se muestran nada de acuerdo y poco de acuerdo respectivamente. Por su parte, un $18,9 \%$ se muestra bastante de acuerdo y un $24,5 \%$ no se posiciona (ni de acuerdo ni en desacuerdo, figura 5).

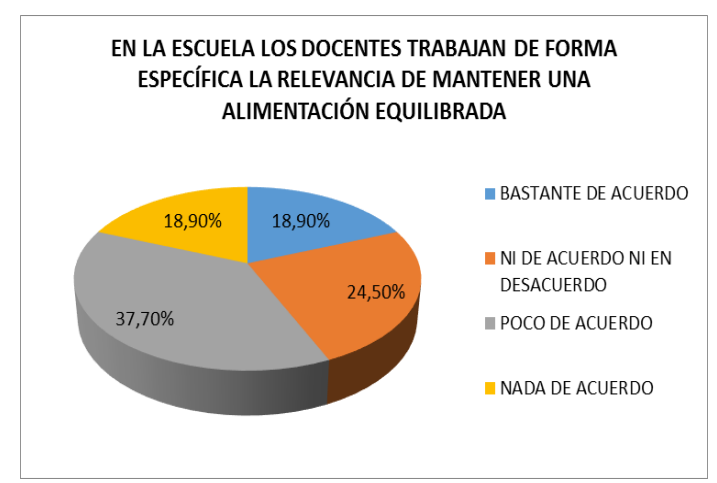

Figura 5. En la escuela los docentes trabajan de forma específica la relevancia de mantener una alimentación equilibrada

Un $28,3 \%$ y un $15,1 \%$ se muestran poco de acuerdo y nada de acuerdo, mientras que un $18,9 \%$ y un $1,9 \%$ se muestran bastante de acuerdo y muy de acuerdo con las posibilidades de iniciativas para la concienciación a las familias, sobre una alimentación sana (figura 6). 


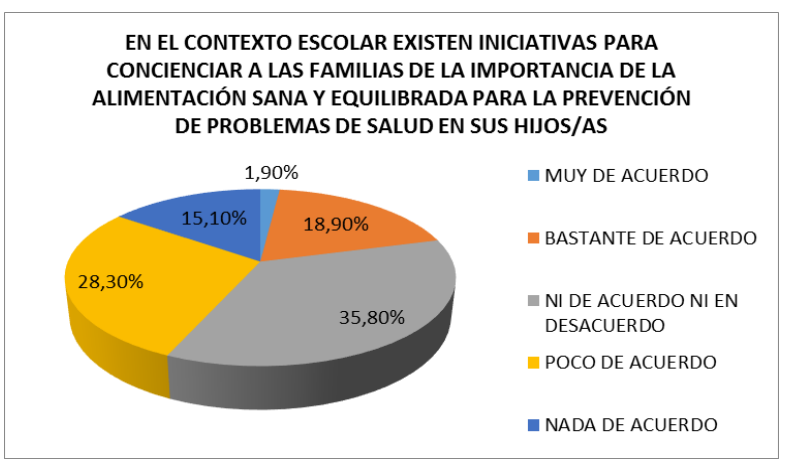

Figura 6. En el contexto escolar existen iniciativas para concienciar a las familias de la importancia de la alimentación sana y equilibrada para la prevención de problemas de salud en sus hijos

En las siguientes gráficas se puede observar que el alumnado universitario tiene una concepción de la EpS más transversal y globalizadora que fragmentada y vinculada a determinadas áreas curriculares en el tratamiento didáctico de la EpS.

El único que caso que concita una mayor conectividad epistemológica y conceptual con los valores y actitudes vinculadas con la EpS es la Educación Física. Así, una amplia mayoría considera que la cuestiones de EpS se trabajan más adecuadamente en las clases de Educación Física. En concreto, un $18,9 \%$ (muy de acuerdo) y un $49,1 \%$ (bastante de acuerdo). Únicamente una minoría de estudiantes no está de acuerdo con el valor pedagógico de vinculación de la Educación Física con la EpS (11,3\%, figura 7).

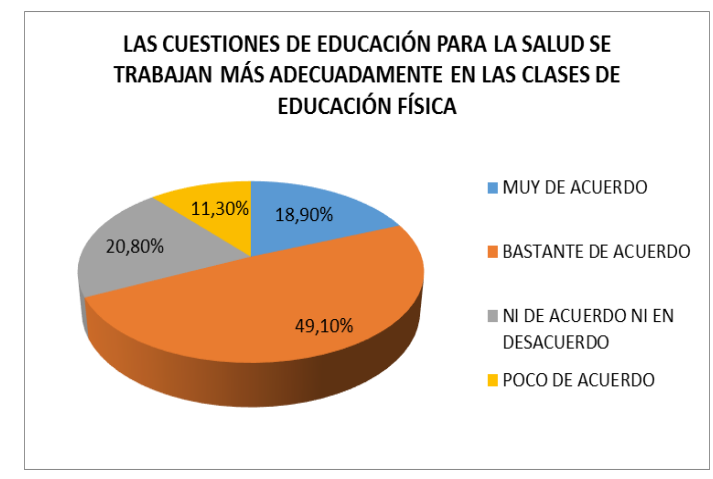

Figura 7. Las cuestiones de EpS se trabajan más adecuadamente en las clases de Educación Física

Los porcentajes cambian cuando se pregunta por la asignatura de Ciencias Naturales, y es que únicamente un 30,2\% del alumnado universitario se muestra favorable a considerar estas clases como espacios privilegiados para el desarrollo de la EpS. Por su parte, un $15,1 \%$ está nada de acuerdo y un $22,6 \%$ se muestra poco de acuerdo. También llama la atención de un $32,1 \%$ de estudiantes que no se inclina hacia ninguna posición (figura 8).

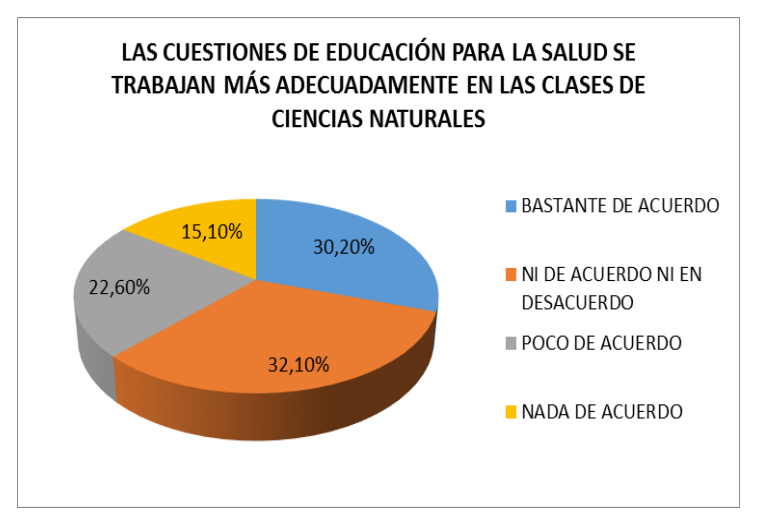

Figura 8. Las cuestiones de Educación para la Salud se trabajan más adecuadamente en las clases de Ciencias Naturales

En el caso de la asignatura de Ciencias Sociales, hay un rechazo mayor respecto a lo que podría ser un cierto parentesco conceptual y de trabajo pedagógica para el abordaje de núcleos temáticos y valores asociadas a la EpS. Se observa que un 45,3\% se muestra poco de acuerdo y un $43,4 \%$ está nada de acuerdo con la posibilidad de trabajar EpS en clases de Ciencias Sociales (figura 9).

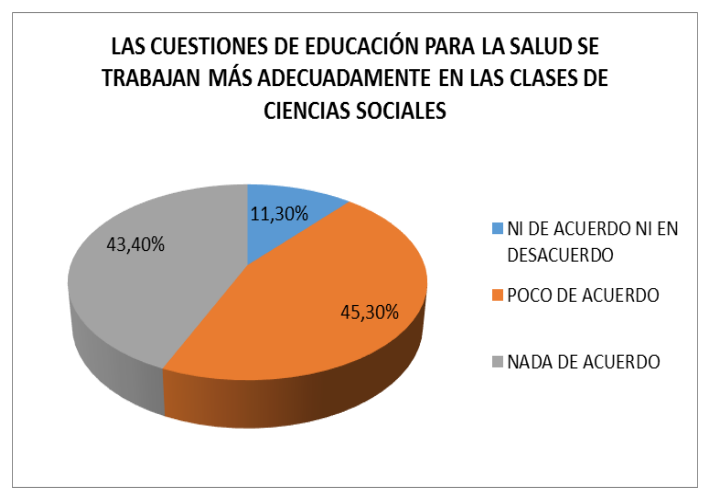

Figura 9. Las cuestiones de Educación para la Salud se trabajan más adecuadamente en las clases de Ciencias Sociales

Un efecto, resulta muy interesante conocer la inexistencia de cualquier tipo de apoyo a la posible vinculación entre Ciencias Sociales y EpS desde la perspectiva de la atención escolar en Educación Primaria. Un $37,7 \%$ se muestra poco de acuerdo, y un $9,4 \%$ está nada de acuerdo con la realidad de que se estudien en el contexto escolar conceptos relacionados con la EpS.

Un $17 \%$ se muestra bastante de acuerdo y únicamente un $1,9 \%$ está muy de acuerdo con esta opción (figura 10). 


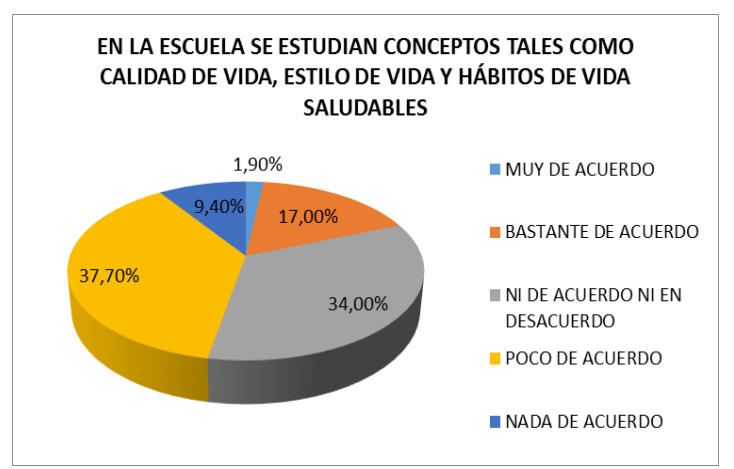

Figura 10. En la escuela se estudian conceptos tales como calidad de vida, estilo de vida y hábitos de vida saludables

Un $45,3 \%$ se muestra poco de acuerdo hacia la afirmación de que la escuela trabaja de forma transversal la EpS en todas las materias curriculares. Únicamente existe un $1,9 \%$ alumnado universitario que cree que la institución escolar sí aborda de forma transversal la EpS en las aulas y centros educativos (figura 11).

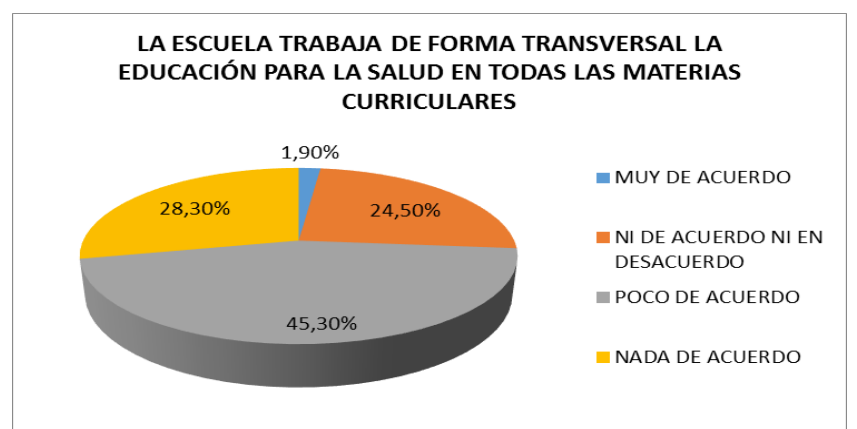

Figura 11. La escuela trabaja de forma transversal la Educación para la Salud en todas las materias curriculares

De forma específica, un $52,8 \%$ de los estudiantes se muestra muy de acuerdo con la afirmación de que la actividad física es primordial para la promoción activa de hábitos de vida saludables en el contexto escolar, y también un 37,7\% afirma estar bastante de acuerdo con ello. Únicamente un $1,9 \%$ se muestra poco de acuerdo con esta consideración (figura 12).

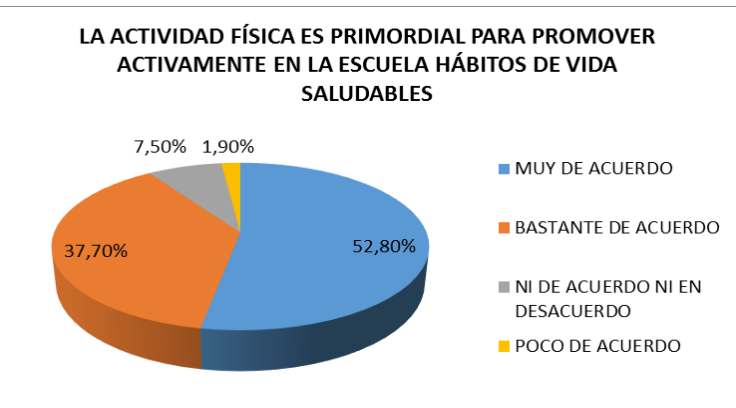

Figura 12. La actividad física es primordial para promover activamente en la escuela hábitos de vida saludable
Así un 1,9\% se muestra muy de acuerdo con que haya diferencias de género a la hora de fomentar la EpS, y un 11,0\% bastante de acuerdo, mientras que un $20,8 \%$ dice que está poco de acuerdo y un $24,5 \%$ se muestra nada de acuerdo. Resulta llamativo el porcentaje de alumnado indefinido en la opción de ni de acuerdo ni en desacuerdo (figura 13).

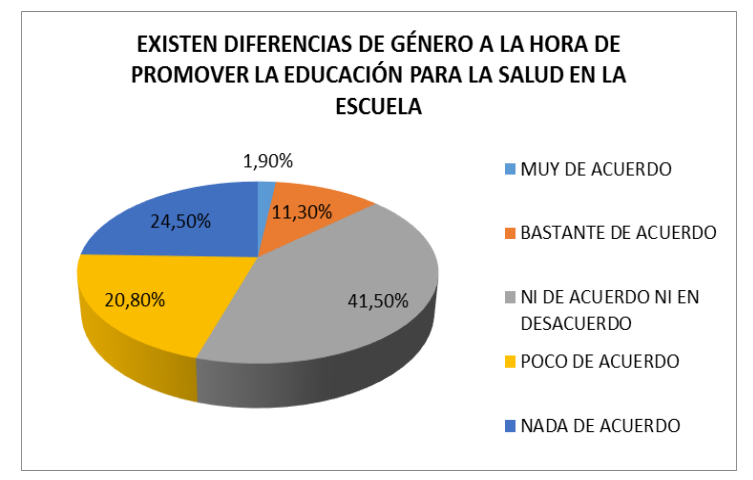

Figura 13. Existen diferencias de género a la hora de promover la EpS en la escuela

La figura 14 muestra resultados más o menos similares sobre la posibilidad de que el alumnado tenga hábitos de vida saludables diferenciados a causa de su género. Así, un 1,9\% está muy de acuerdo con ello, y también un $15,1 \%$ se muestra bastante de acuerdo con esta consideración.

Por el contrario, una mayoría de estudiantes universitarios niegan la realidad de una perspectiva de género en relación a la configuración de hábitos de vida saludables En concreto un 26,4\% se muestra poco de acuerdo y un $18,9 \%$ afirma estar nada de acuerdo. Al igual que en la figura 13, destaca el porcentaje elevado de estudiantes que se muestran ni de acuerdo ni en desacuerdo, un $37,7 \%$.

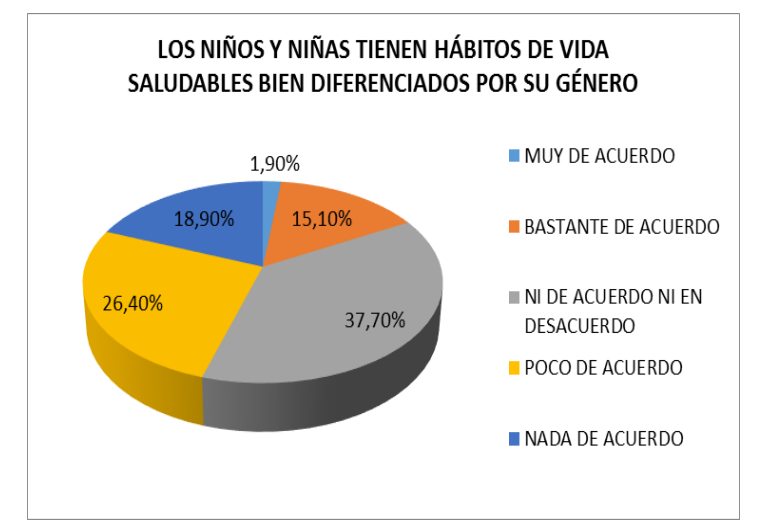

Figura 14. Los niños tienen hábitos de vida saludables bien diferenciados por su género

Se pone de relieve la existencia de un $43,3 \%$ de alumnado universitario que está poco de acuerdo con la realidad de la formación específica en EpS por 
parte del profesorado en activo en Educación Primaria. Igualmente, un 26,4\% afirma estar nada de acuerdo. Muy pocos estudiantes plantean lo contrario (figura 15).

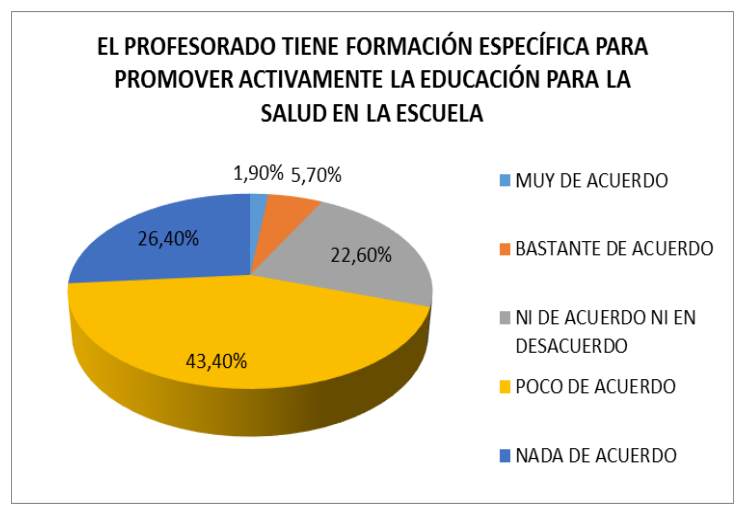

Figura 15. El profesorado tiene formación específica para promover activamente la EpS en la escuela

Finalmente, la figura 16 muestra una amplia mayoría que afirma estar muy de acuerdo con que debería existir una asignatura de EpS en el currículum escolar. Un $22,6 \%$ está bastante de acuerdo con esta consideración y un $69,8 \%$ se muestra muy de acuerdo.

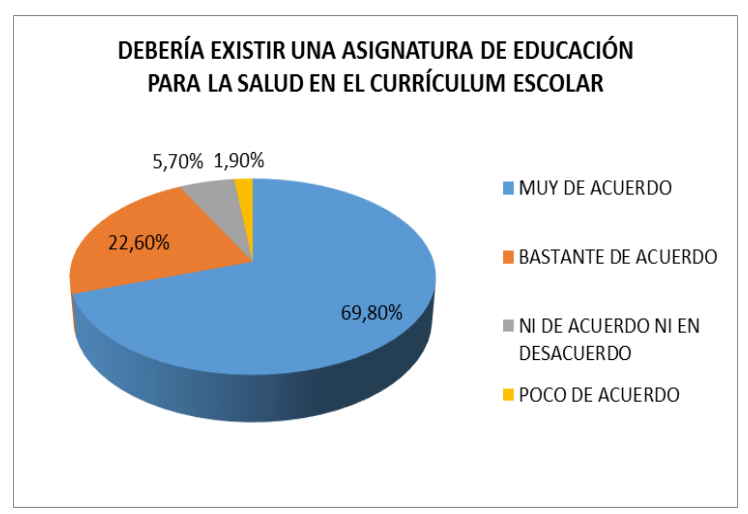

Figura 16. Debería existir una asignatura de EpS en el currículum escolar

\section{CONCLUSIONES Y DISCUSIÓN}

Este estudio muestra la existencia de un grupo de estudiantes críticos con el papel y funciones de la escuela en la EpS, Las instituciones escolares han estado históricamente preocupadas de las dimensiones transmisivas y más bien cognitivistas del conocimiento científico, y poco ocupadas por el bienestar y la integralidad del alumnado como un todo. Es decir, que el bienestar y la calidad de vida han sido aspectos escasamente abordados en los contextos educativos formales.

Por otro lado, el alumnado universitario se muestra en este estudio muy crítico con las posibilidades pedagógicas de que existan iniciativas ciertas para la concienciación a las familias del valor relevante de la alimentación sana y equilibrada para la prevención de problemas de salud en sus hijos. En todo caso, se ha hallado una división más o menos acentuada, aunque se valora la existencia un escepticismo claro por parte de los estudiantes sobre esta cuestión.

En este sentido, parece que se impone la idea de un tratamiento más bien deficitario y reduccionista de los conceptos de calidad de vida, así como de estilos de vida en relación con la EpS.

Uno de los resultados más relevantes del estudio es la negación del carácter transversal que adopta la escuela actual en relación con el tratamiento didáctico de la EpS.

Esto lleva a plantearse la necesidad de formar a los propios estudiantes en estos temas, siendo conocedores de la escasez de referencias temáticas en el plan de estudios del Grado de Educación Primaria, en el contexto de la Facultad de Ciencias de la Educación de la Universidad de Málaga; y siendo conscientes de que esta situación se repite en la mayoría de titulaciones de Ciencias de la Educación en el panorama universitario autonómico de Andalucía.

Lo que sí se observa es un posicionamiento casi unánime sobre la relevancia de la actividad física como soporte básico para la promoción activa en las escuelas y organizaciones escolares de hábitos de vida saludables. Así, del presente estudio se desprende la vinculación necesaria de la Educación Física, de actividad física con una adecuada relación conceptual e incluso procedimental sobre el fomento proactivo de hábitos de vida saludables, que bien se podrían inculcar en las titulaciones de Ciencias de la Educación. Se podría inferir que esto puede ser abordado en materias específicas y optativas del itinerario de Educación Física que escogen los estudiantes del Grado de Educación Primaria a partir del $3^{\circ}$ curso.

Otra conclusión que se desprende de este estudio es la idea de una cierta confusión y división entre el alumnado universitario sobre la existencia de diferencias de género a la hora de fomentar la EpS en la escuela.

En otro orden de cosas, existe una abrumadora mayoría de estudiantes que plantea que el profesorado tiene una escasa formación especializada EpS, lo cual puede redundar en iniciativas pedagógicas más bien escasas en esta materia en la realidad escolar.

Esto no es contradictorio en relación a la crítica que realizan los estudiantes universitarios sobre el 
ineficaz tratamiento didáctico del EpS en el espacio escolar, y, sobre todo, con la ausencia de una visión o enfoque más bien interdisciplinar o transversal de la EpS. Por tanto, los estudiantes del Grado de Educación Primaria tienen una actitud francamente positiva hacia la EpS y plantean la existencia de una asignatura específica de EpS en el currículum escolar. Esto se podría incluso extrapolar a la posibilidad de una materia formativa universitaria, pero no ha sido objeto de cuestionamiento y no podemos afirmar nada a ese respecto.

Así, los resultados son coherentes con Giraldo, Toro, Macías, Garcés, Andrés y Palacio (2010) cuando se plantean que la promoción de la salud es una estrategia pedagógica inclusiva que requiere la implementación de todo tipo de acciones vinculadas a la puesta en práctica de hábitos de vida saludables. Para ello, es fundamental la formación en EpS desde el contexto de formación inicial del profesorado, de tal forma que exista un vínculo teórico-práctico $\mathrm{y}$, sobre todo, de educación en valores que faculte a los futuros docentes el desarrollo de iniciativas didácticas que surtan efecto en la población infantil y juvenil de nuestro país.

Los tratamientos didácticos globalizadores de carácter interdisciplinar son sumamente positivos y necesarios tanto en la educación obligatoria como en la propia formación inicial del profesorado. El problema se sitúa en el sentido, orientación e impronta que pueda adoptar el enfoque transversal o específico si se llega a la conclusión de que es mejor convertir a la EpS en una materia o área curricular específica. Nuestro estudio apunta a que pudiera ser una opción, pero lo cierto es que lo más plausible es impulsar la formación en EpS y respaldar un enfoque interdisciplinar, intercultural y competencial en el abordaje temático y actitudinal del EpS para seguir mejorando la calidad de vida de las personas, tanto desde la perspectiva de los estilos de vida saludables en una visión micro como en una visión macro y de intervención pública y comunitaria.

No se trata únicamente de un proceso de mera transmisión de información, sino todo lo contrario, la opción de que los estudiantes que se están preparando para ser buenos, o mejor dicho, excelentes educadores, se preocupen por impregnar sus actitudes de valores favorables a la adquisición de hábitos de vida saludable para así poder conciencia y sensibilizar mejor a sus futuros estudiantes. El concepto de EpS añade precisión al concepto de sostenibilidad al atender a la singularidad y a la diversidad de modelos y estilos de vida asociados a la globalización cultural (Rochon, 2001). Por tanto, existe distintas visiones de EpS que pueden converger en la perspectiva intercultural e inclusiva en el tratamiento didáctico de los hábitos de vida saludables comunes en toda la humanidad.

Finalmente, es necesario advertir que la existencia de un alto porcentaje de opiniones ambigua lleva a tratar con cautela y prudencia los resultados obtenidos. Incluso podría sospecharse que este alumnado universitario no recuerda bien su experiencia y/o trayectoria escolar, o bien no quiere posicionarse en relación con sus actitudes hacia la escuela como promotora o no de hábitos de vida saludable.

\section{REFERENCIAS}

Cubero, J., Calderón, M., Costillo, E., y Ruiz, C. (2011). La Educación para la Salud en el Espacio Europeo de educación superior. Publicaciones, 41, 51-63.

Gavidia, V. (2001). La transversalidad y la escuela promotora de salud. Revista Española de Salud Publica, 75(6), 505516.

Giraldo, A., Toro, M. Y., Macías, A. M., Garcés, V., Andrés, C., y Palacio, S. (2010). La promoción de la salud como estrategia para el fomento de estilos de vida saludables. Hacia la Promoción de la Salud, 15(1), 128-143.

González de Haro Mª D. (2008). La Educación para la Salud: "asignatura pendiente para la escuela", XXI Revista de Educación. 10, 123-136.

Macías, A. I., Gordillo, L. G., y Camacho, E. J. (2012). Hábitos alimentarios de niños en edad escolar y el papel de la Educación para la Salud. Revista chilena de nutrición, 39(3), 40-43.

Muñoz M. y Cabieses B. (2008). Universidades y pro- moción de la salud: ¿cómo alcanzar el punto de encuentro? Revista Panameña de Salud Pública, 24 (2), 139-146.

Rochon, A. (2001). Educación para la Salud. Guía práctica para realizar proyectos. Barcelona: Masson. 